\title{
Extracellular vesicles and aging
}

\author{
Paul D. Robbins \\ Department of Molecular Medicine and the Center on Aging, the Scripps Research Institute, Jupiter, Florida, USA \\ Correspondence to: Paul D. Robbins. Department of Molecular Medicine, The Scripps Research Institute, 130 Scripps Way, \#3B3, Jupiter, Florida \\ 33458, USA. Email: probbins@scripps.edu.
}

\begin{abstract}
Aging and the chronic diseases associated with aging place a tremendous burden on our healthcare system. As our world population ages dramatically over the next decades, this will only increase. Hence, there is a great need to discover fundamental mechanisms of aging to enable development of strategies for minimizing the impact of aging on our health and economy. There is general agreement that cell autonomous mechanisms contribute to aging. As cells accrue damage over time, they respond to it by triggering individual cell fate decisions that ultimately disrupt tissue homeostasis and thus increase risk of morbidity. However, there are numerous lines of evidence, including heterochronic parabiosis and plasma transfer, indicating that cell non-autonomous mechanisms are critically important for aging as well. In addition, senescent cells, which accumulate in tissues with age, can display a senescence-associated secretory phenotype (SASP) that contributes to driving aging and loss of tissue homeostasis through a non-cell autonomous mechanism(s). Given the diverse roles of blood-borne extracellular vesicles (EVs) in modulating not only the immune response, but also angiogenesis and tissue regeneration, they likely play a key role in modulating the aging process through cell non-autonomous mechanisms. The fact that senescent cells release more EVs and with a different composition suggests they contribute to the adverse effects of senescence on aging. In addition, the ability of EVs from functional progenitor cells to promote tissue regeneration suggests that stem cell-derived EVs could be used therapeutically to extend healthspan. This review focuses on the potential roles of EVs in aging, the potential of EV-based therapeutic applications for extending healthspan and the potential for use of circulating EVs as biomarkers of unhealthy aging.
\end{abstract}

Keywords: Aging; extracellular vesicles (EVs); mesenchymal stem cells (MSCs)

Received: 18 October 2017; Accepted: 06 December 2017; Published: 19 December 2017.

doi: $10.21037 /$ sci.2017.12.03

View this article at: http://dx.doi.org/10.21037/sci.2017.12.03

\section{Aging}

It is estimated that in the next 20 years, the number of individuals in the United States over the age of 65 will double, numbering more than 70 million individuals. Unfortunately, as we age there is an unavoidable and progressive loss of the ability to maintain tissue homeostasis under stress and an attrition of functional reserve. As a consequence, the incidence of numerous debilitating diseases increases nearly exponentially with age, including cardiovascular disease, neurodegeneration, diabetes, osteoarthritis, and osteoporosis. Over $90 \%$ of individuals $>65$ years of age have at least one chronic disease, while $>70 \%$ have at least two. These chronic diseases account for
$75 \%$ of our healthcare costs, amounting to approximately $\$ 3$ trillion in costs last year alone. Indeed, chronic diseases of the elderly are the greatest healthcare burden in the United States and seriously impact the quality of life of a large segment of the population. Thus, there is a significant need to understand mechanisms driving aging and to develop novel therapeutics. Given the diverse roles of blood-borne EVs in modulating not only the immune response, but also angiogenesis and tissue regeneration, they likely play a key role in modulating the aging process. This review focuses on the role EVs could play in aging, their therapeutic application for extending healthspan and their potential for use as biomarkers of unhealthy aging. 


\section{Mechanisms underlying aging}

Aging is a complex process involving a number of different pathways with both genetic and environmental components (1-5). Aging is thought to arise, in part, as a consequence of the accumulation of stochastic molecular and cellular damage. The precise nature of the damage responsible for aging-related degeneration remains poorly defined, but likely consists of mitochondrial dysfunction, elevated ROS, telomere attrition, changes in nuclear structure, accumulation of genetic mutations, or DNA, protein and membrane damage. Biological processes that underlie aging phenotypes and are also active at sites of etiology of most chronic diseases include: (I) chronic, low-grade, "sterile" inflammation; (II) macromolecular and organelle dysfunction resulting in changes in level or function of proteins, carbohydrates, lipids, mitochondria and DNA; (III) stem cell and progenitor cell dysfunction; and (IV) increased senescent cell burden. These four processes are linked in that interventions targeting one process also attenuate the others. For example, oxidative DNA damage increases stochastically in cells in different tissues, likely driven in part by increased mitochondrial ROS, resulting in the induction of cellular senescence. These senescent cells accumulate with age at sites of pathogenesis in chronic diseases $(6,7)$. Reduction of the senescent cell burden can lead to reduced inflammation, decreased macromolecular dysfunction, and enhanced function of progenitors (8-10). Also, adult stem cells become dysfunctional with evidence of senescence with age, likely driven by macromolecular and organelle dysfunction (11). These four biological processes are also the key components of the seven pillars of aging, defined as adaption to stress, epigenetics, inflammation, macromolecular damage, metabolism, proteostasis and stem cells and regeneration.

\section{Autonomous and non-autonomous mechanisms of aging}

There is compelling evidence to support the hypothesis that the underlying cause of aging is the cell autonomous, time-dependent accumulation of stochastic damage to cells, organelles and macromolecules. However, it is also clear from heterochronic parabiosis (12-17) and serum transfer $(17,18)$ studies that cell non-autonomous mechanisms play important roles in suppressing or driving degenerative changes that arise as the consequence of spontaneous, stochastic damage. For example, using heterochronic parabiosis, it was demonstrated that factors in young blood rejuvenate certain cell types and tissues in old mice (12-17). These anti-geronic factors in young serum include GDF-11 and oxytocin (19). Treatment of mice with rGDF-11 $(15,16,20)$, similar to heterochronic parabiosis, has rejuvenating effects on skeletal muscle, heart and brain, although these results are still controversial $(21,22)$. Furthermore, factors in umbilical cord, but not adult, plasma function as synaptic plasticity-promoting proteins with TIMP2 demonstrated to increase hippocampaldependent cognition (23). Conversely, factors in old blood can drive aging of certain cell types and tissues in young mice. These blood-borne pro-geronic factors include the chemokine CCL-11 (24) and $\beta-2$ microglobulin (25). In addition to these identified geronic factors, it is likely there are other circulating factors that also play key, cell nonautonomous roles in aging. Indeed, it is likely a combination of loss of anti-geronic factors and an increase in pro-geronic factors that drives aging. Given that almost all cell types release EVs, including stem/progenitor cells and senescent cells, it is likely that subsets of blood-borne EVs play key roles as both anti- and pro-geronic factors.

\section{Cellular senescence}

Senescence is a cell fate that involves loss of proliferative potential of normally replication-competent cells with associated resistance to cell death through apoptosis and generally increased metabolic activity. Frequently, senescent cells develop a senescence-associated secretory phenotype (SASP) characterized by increased release of proinflammatory cytokines and chemokines, tissue-damaging proteases, factors that can impact stem and progenitor cell function, hemostatic factors, and growth factors (8). Markers of senescent cells include increases in expression of the cell cycle regulators, p16INK4A and p21Cip1, of SASP factors (e.g., IL-6, IL-8, monocyte chemoattractant protein-1, plasminogen-activated inhibitor-1, and many others), increased senescence-associated $\beta$-galactosidase (SA- $\beta$ gal) activity, senescent-associated distension of satellites (SADS), and telomere-associated DNA damage foci (TAFs), among others. Senescent cells that express the SASP thus can have substantial pathologic effects. In support of an important role for senescence in aging, selective killing of p16INK4a-positive senescent cells extended healthspan in transgenic mouse models (INKATTAC and p16-3MR mice) of accelerated aging 
(26-30). Importantly, clearing senescent cells from aged INK-ATTAC mice improved age-related changes in metabolic function (9). Subsequently, it was demonstrated that chronic clearance of p16INK4a-positive cells in adult mice extends the median lifespan of naturally aged mice (27). Clearance of senescent cells in versions of this genetic model (INK-ATTAC and 3MR mice) or treating mice with novel senolytics extended healthspan $(31,32)$, restored vascular reactivity (33), stabilized atherosclerotic plaques (34), improved pulmonary function (35), alleviated osteoarthritis (28), improved fatty liver disease (36) and improved lung function in a pulmonary fibrosis model (35). Conversely, injecting senescent cells is able to drive agerelated diseases such as osteoarthritis (37). Thus, the increase in cellular senescence that occurs with aging appears to play a major role in driving life-limiting, agerelated diseases $(8,29,30,38-40)$. As discussed below, senescent cells release more EVs with a different composition, suggesting that EVs should be considered part of the SASP, important for conferring the adverse effects of senescent cells on aging.

\section{Stem cells and aging}

A characteristic of aging is the loss of regenerative capacity, leading to an impaired ability to respond to stress and therefore increased morbidity and mortality. This has led to the hypothesis that aging is caused, in part, by the loss of functional adult stem cells necessary for maintaining tissue homeostasis. Indeed, mice greater than 2 years of age have a significant reduction in the number and proliferative capacity of different adult stem cell types. For example, there are age-related changes in bone marrow-derived mesenchymal stem cells (BM-MSCs) including loss of proliferation and differentiation potential and increased senescence. Similarly, MSCs derived from the bone marrow of patients with Hutchinson-Gilford Progeroid Syndrome, a disease of accelerated aging, are defective in their ability to differentiate (41). In addition, muscle derived stem/progenitor cells (MDSPC) are adversely affected in accelerated and natural aged mice, displaying loss of proliferation and ability to differentiate in culture $(11,42)$. Importantly, this dysfunction was demonstrated to directly contribute to age-related degenerative changes since intraperitoneal injection of only 106 functional, young MDSPCs was sufficient to extend healthspan and lifespan in two different mouse models of accelerated aging (11). Only a few of the injected, labeled MDSPCs were found in different tissues with no evidence of differentiation, suggesting that the therapeutic effect of MDSPCs is likely mediated by secreted factors that act systemically in a cell nonautonomous manner. Consistent with this hypothesis, coculture of young, functional MDSPCs with old MDSPCs in a transwell system resulted in improvement in the ability of the old MDSPCs to proliferate and differentiate (11). At least part of this activity co-purifies with EVs.

Injection of young, functional BM-MSCs into rats has been shown to extend their lifespan. Ubiquitously located throughout the body, MSCs can act locally through chemotactic-induced migration from the perivascular niches in response to stress or injury as well as systemically through the secretion of various soluble factors such as chemokines, cytokines and extracellular vesicles (EVs). Tasked with maintaining the HSC niche through the regeneration of an extracellular matrix comprised of osteoblasts, adipocytes and endothelial cells (43-45), MSCs also maintain tissue homeostasis through modulating HSC function. In addition, MSCs are vital in maintaining blood vessel integrity through promotion of angiogenesis and thus are essential for systemic wound healing and tissue regeneration. Lastly, MSCs have a profound capacity to modulate the immune system, therefore modulating the immune response to stress and injury by regulating the proinflammatory response of macrophages and prohibiting lymphocyte proliferation (46). MSCs derived from old mice are defective in their ability to differentiate and undergo senescence more rapidly in culture.

\section{EVs}

EVs are comprised of both microvesicles, released from the plasma membrane by shedding, and nanovesicles or exosomes, generated by reverse budding of multivesicular bodies (MVBs) $(47,48)$. These different types of EVs are characterized predominantly by their size, with exosomes ranging from 30 to $100 \mathrm{~nm}$ and microvesicles usually being larger than $100 \mathrm{~nm}$. Although their contents likely differ, both small and large EVs are enriched for a subset of diverse proteins, lipids, messenger RNAs (mRNAs), and non-coding RNAs (ncRNAs), such as miRNAs, which are derived from the parental cells. EVs have a variety of reported functions and some of their betterdocumented activities are associated with some form of immune regulation $(47,48)$. EVs from both immune and non-immune cells, such as MSCs and endothelial cells, contribute to antigen-specific and non-specific immune 
regulation (47-49). Depending upon the context and vesicle type, EVs can stimulate or suppress the immune responses to infections with viruses and microbial pathogens as well as cancer.

EVs derived from stem cells also have significant ability to repair damaged tissue (50). For example, EVs derived from marrow or adipose MSCs affect the phenotype and induce healing of many different tissue and cell types, including liver (51), heart (52), pulmonary epithelial cells and kidney $(53,54)$ as well as promote angiogenesis $(55,56)$. Consistent with these regenerative capacities of stem cell EVs, a recent study demonstrated that implantation of healthy hypothalamic stem/progenitor cells into the hypothalamus leads to the slowing of ageing (57). Moreover, it was demonstrated that the functional hypothalamic stem/ progenitor cells release exosomes into the cerebral spinal fluid that likely contribute to slowing aging through transfer of miRNAs (57). Conversely, it has been demonstrated that senescent cells release more EVs and with a different composition (58-60), likely contributing to the SASP. Taken together, these results suggest that functional stem/ progenitor cell-derived EVs are able to extend healthspan and lifespan whereas senescent cell-derived EVs could function as pro-geronic factors.

\section{Circulating, blood-borne vesicles}

EVs are found in blood and circulate throughout the body, presumably serving as a form of cell-to-cell communication at a distance. Given that EVs contain RNA, proteins and lipids derived from the cell of origin, components of circulating EVs are being used as markers of disease. For examples, tumor associated proteins such as EGF-R (glioblastoma) (61) or oncogene mRNAs (62) have been found in cerebral spinal fluid or blood-derived EVs respectively whereas the protein glypican-1 is found in circulating EVs from patients with pancreatic cancer (63). Also, circulating EVs have important biological activities $(47,48)$. Serum-derived EVs from mice bearing tumors were able to suppress tumor antigen-specific responses (64-66). Similarly, intradermal immunization with a specific antigen resulted in the presence of MHC Class II+ EVs in the serum able to suppress antigen-specific immune responses in a mouse delayed type hypersensitivity (DTH) footpad model (67). More recently, it was demonstrated that EVs from human serum can promote vascular remodeling and prevent muscle damage in a mouse model of acute hind limb ischemia (68).
Taken together, these observations strongly suggest that EVs play important roles in immune regulation and tissue regeneration. More importantly, these results suggest that circulating EVs in the blood could contribute to cell nonautonomous mechanisms of aging. Indeed, given that EVs are important for cell-to-cell communication between neighboring cells and cells at a distance, transferring not only RNA, but also proteins, lipids and metabolites, they are well-positioned to play key pro- and anti-geronic roles with aging.

\section{EVs as biomarkers of aging}

EVs can act as a biomarker, specifying the progression of the disease state of the cells in which they originate. For example, EVs from the serum of aged rats have been shown to have reduced CD63 and increased acetylcholinesterase (AChE) levels compared to young controls. Interestingly, exercise in the aged animals altered the CC63 and AChE levels. The miR-183 cluster, comprised of miR-96, miR-182 and miR-183, increases with age, at least in EVs derived from bone marrow (69). Interestingly, transfection of a miRNA-183-5p mimic was shown to reduce cell proliferation and increase senescence in bone marrow stem cells, suggesting the bone marrow EVs from aged animals could suppress osteogenesis. In addition, increased levels of proBDNF and BDNF were found in circulating L1CAM+EVs, derived from neuronal cells (70). Individuals with higher EV BDNF levels had slower walking speeds (70). In prostate cancer patients on dietary protein restriction, an increase in the levels of leptin receptor in total plasma EVs and, in particular, the L1CAM+EV subset was observed (71). There also was a change in the phosphorylation status of the insulin receptor signal transducer protein IRS1 in L1CAM+ EVs (71). These results suggest that protein restriction could improve insulin and leptin sensitivity. In a recent study, the levels of circulating EVs in plasma were shown to decrease in a cross-sectional and longitudinal study. Here it also was demonstrated that plasma EVs from older individuals had increased MHC-II expression on monocytes and were more readily internalized by B cells (72). This uptake of the plasma EVs results in activation of not only B cells, but also monocytes (72). Thus circulating EVs in aged individuals likely can modulate the immune response.

\section{Summary}

Given the observations that heterochronic parabiosis 
and plasma transfer can slow aging in old animals and accelerate aging in young animals, circulating factors act as anti and pro-geronic factors to modulate aging. The fact that EVs are released by many cell types in vivo and play important roles in cell-to-cell communication make EVs the perfect candidates for key geronic factors. Indeed, blood-borne EVs have been shown to modulate not only the immune response, but also promote angiogenesis and tissue regeneration. In addition, cellular senescence contributes to driving aging through release of soluble factors, as demonstrated by the fact clearance of senescent cells extends general healthspan. Since senescent cells release more EVs than non-senescent cells in culture and potentially in vivo, EVs likely are part of the SASP, contributing to the age-related pathologies driven by cellular senescence. Conversely, the ability of EVs from functional progenitor cells to promote tissue regeneration suggests that stem cell-derived EVs could be used therapeutically to extend healthspan. Taken together, there is substantial circumstantial evidence that EVs play key roles in aging and that regenerative EVs could be used to extend healthy aging. Finally, given the likely role of EVs in aging, components of EVs, in particular EV subsets such as L1CAM+ EVs, could be developed as biomarkers of unhealthy aging.

\section{Acknowledgements}

Funding: This work was supported in part by P01 AG43376 from the National Institutes of Health.

\section{Footnote}

Conflicts of Interest: The author has no conflicts of interest to declare.

\section{References}

1. Kirkwood TB. Understanding ageing from an evolutionary perspective. J Intern Med 2008;263:117-27.

2. Passos JF, von Zglinicki T, Kirkwood TB. Mitochondria and ageing: winning and losing in the numbers game. Bioessays 2007;29:908-17.

3. Vijg J, Campisi J. Puzzles, promises and a cure for ageing. Nature 2008;454:1065-71.

4. Campisi J, Vijg J. Does damage to DNA and other macromolecules play a role in aging? If so, how? J Gerontol A Biol Sci Med Sci 2009;64:175-8.
5. Hoeijmakers JH. DNA damage, aging, and cancer. N Engl J Med 2009;361:1475-85.

6. Kirkland JL. Translating the science of aging into therapeuticiInterventions. Cold Spring Harb Perspect Med 2016;6:a025908.

7. Krishnamurthy J, Torrice C, Ramsey MR, et al. Ink4a/ Arf expression is a biomarker of aging. J Clin Invest 2004;114:1299-307.

8. Tchkonia T, Zhu Y, van Deursen J, et al. Cellular senescence and the senescent secretory phenotype: therapeutic opportunities. J Clin Invest 2013;123:966-72.

9. Xu M, Palmer AK, Ding H, et al. Targeting senescent cells enhances adipogenesis and metabolic function in old age. Elife 2015;4:e12997.

10. Zhu Y, Armstrong JL, Tchkonia T, et al. Cellular senescence and the senescent secretory phenotype in agerelated chronic diseases. Curr Opin Clin Nutr Metab Care 2014;17:324-8.

11. Lavasani M, Robinson AR, Lu A, et al. Muscle-derived stem/progenitor cell dysfunction limits healthspan and lifespan in a murine progeria model. Nat Commun 2012;3:608.

12. Conboy IM, Conboy MJ, Wagers AJ, et al. Rejuvenation of aged progenitor cells by exposure to a young systemic environment. Nature 2005;433:760-4.

13. Conboy IM, Rando TA. Heterochronic parabiosis for the study of the effects of aging on stem cells and their niches. Cell Cycle 2012;11:2260-7.

14. Conboy MJ, Conboy IM, Rando TA. Heterochronic parabiosis: historical perspective and methodological considerations for studies of aging and longevity. Aging Cell 2013;12:525-30.

15. Loffredo FS, Steinhauser ML, Jay SM, et al. Growth differentiation factor 11 is a circulating factor that reverses age-related cardiac hypertrophy. Cell 2013;153:828-39.

16. Sinha M, Jang YC, Oh J, et al. Restoring systemic GDF11 levels reverses age-related dysfunction in mouse skeletal muscle. Science 2014;344:649-52.

17. Rebo J, Mehdipour M, Gathwala R, et al. A single heterochronic blood exchange reveals rapid inhibition of multiple tissues by old blood. Nat Commun 2016;7:13363 .

18. Villeda SA, Plambeck KE, Middeldorp J, et al. Young blood reverses age-related impairments in cognitive function and synaptic plasticity in mice. Nat Med 2014;20:659-63.

19. Elabd C, Cousin W, Upadhyayula P, et al. Oxytocin is an age-specific circulating hormone that is necessary for muscle maintenance and regeneration. Nat Commun 
2014;5:4082

20. Katsimpardi L, Litterman NK, Schein PA, et al. Vascular and neurogenic rejuvenation of the aging mouse brain by young systemic factors. Science 2014;344:630-4.

21. Zimmers TA, Jiang Y, Wang M, et al. Exogenous GDF11 induces cardiac and skeletal muscle dysfunction and wasting. Basic Res Cardiol 2017;112:48.

22. Hammers DW, Merscham-Banda M, Hsiao JY, et al. Supraphysiological levels of GDF11 induce striated muscle atrophy. EMBO Mol Med 2017;9:531-44.

23. Castellano JM, Mosher KI, Abbey RJ, et al. Human umbilical cord plasma proteins revitalize hippocampal function in aged mice. Nature 2017;544:488-92.

24. Villeda SA, Luo J, Mosher KI, et al. The ageing systemic milieu negatively regulates neurogenesis and cognitive function. Nature 2011;477:90-4.

25. Smith LK, He Y, Park JS, et al. beta2-microglobulin is a systemic pro-aging factor that impairs cognitive function and neurogenesis. Nat Med 2015;21:932-7.

26. Baker DJ, Wijshake T, Tchkonia T, et al. Clearance of p16Ink4a-positive senescent cells delays ageing-associated disorders. Nature 2011;479:232-6.

27. Baker DJ, Childs BG, Durik M, et al. Naturally occurring p16(Ink4a)-positive cells shorten healthy lifespan. Nature 2016;530:184-9.

28. Jeon OH, Kim C, Laberge RM, et al. Local clearance of senescent cells attenuates the development of posttraumatic osteoarthritis and creates a pro-regenerative environment. Nat Med 2017;23:775-81.

29. Kirkland JL, Tchkonia T, Zhu Y, et al. The Clinical Potential of Senolytic Drugs. J Am Geriatr Soc 2017;65:2297-301.

30. Kirkland JL, Tchkonia T. Cellular Senescence: A Translational Perspective. EBioMedicine 2017;21:21-8.

31. Zhu Y, Tchkonia T, Pirtskhalava T, et al. The Achilles' heel of senescent cells: from transcriptome to senolytic drugs. Aging Cell 2015;14:644-58.

32. Fuhrmann-Stroissnigg H, Ling YY, Zhao J, et al. Identification of HSP90 inhibitors as a novel class of senolytics. Nat Commun 2017;8:422.

33. Roos CM, Zhang B, Palmer AK, et al. Chronic senolytic treatment alleviates established vasomotor dysfunction in aged or atherosclerotic mice. Aging Cell 2016;15:973-7.

34. Childs BG, Baker DJ, Wijshake T, et al. Senescent intimal foam cells are deleterious at all stages of atherosclerosis. Science 2016;354:472-7.

35. Schafer MJ, White TA, Iijima K, et al. Cellular senescence mediates fibrotic pulmonary disease. Nat Commun
2017;8:14532.

36. Ogrodnik M, Miwa S, Tchkonia T, et al. Cellular senescence drives age-dependent hepatic steatosis. Nat Commun 2017;8:15691.

37. Xu M, Bradley EW, Weivoda MM, et al. Transplanted Senescent Cells Induce an Osteoarthritis-Like Condition in Mice. J Gerontol A Biol Sci Med Sci 2017;72:780-5.

38. LeBrasseur NK, Tchkonia T, Kirkland JL. Cellular Senescence and the Biology of Aging, Disease, and Frailty. Nestle Nutr Inst Workshop Ser 2015;83:11-8.

39. Palmer AK, Tchkonia T, LeBrasseur NK, et al. Cellular Senescence in Type 2 Diabetes: A Therapeutic Opportunity. Diabetes 2015;64:2289-98.

40. Kirkland JL, Tchkonia T. Clinical strategies and animal models for developing senolytic agents. Exp Gerontol 2015;68:19-25.

41. Scaffidi P, Misteli T. Lamin A-dependent misregulation of adult stem cells associated with accelerated ageing. Nat Cell Biol 2008;10:452-9.

42. Lavasani M, Lu A, Thompson SD, et al. Isolation of muscle-derived stem/progenitor cells based on adhesion characteristics to collagen-coated surfaces. Methods Mol Biol 2013;976:53-65.

43. Dimarino AM, Caplan AI, Bonfield TL. Mesenchymal stem cells in tissue repair. Front Immunol 2013;4:201.

44. Caplan AI. MSCs: The Sentinel and Safe-Guards of Injury. J Cell Physiol 2016;231:1413-6.

45. Pittenger MF, Mackay AM, Beck SC, et al. Multilineage potential of adult human mesenchymal stem cells. Science 1999;284:143-7.

46. Singer NG, Caplan AI. Mesenchymal stem cells: mechanisms of inflammation. Annu Rev Pathol 2011;6:457-78.

47. Robbins PD, Dorronsoro A, Booker CN. Regulation of chronic inflammatory and immune processes by extracellular vesicles. J Clin Invest 2016;126:1173-80.

48. Robbins PD, Morelli AE. Regulation of immune responses by extracellular vesicles. Nat Rev Immunol 2014;14:195-208.

49. Théry C, Ostrowski M, Segura E. Membrane vesicles as conveyors of immune responses. Nat Rev Immunol 2009;9:581-93.

50. Zhang B, Yeo RW, Tan KH, et al. Focus on Extracellular Vesicles: Therapeutic Potential of Stem Cell-Derived Extracellular Vesicles. Int J Mol Sci 2016;17:174.

51. Tan CY, Lai RC, Wong W, et al. Mesenchymal stem cell-derived exosomes promote hepatic regeneration in drug-induced liver injury models. Stem Cell Res Ther 
2014;5:76.

52. Lai RC, Arslan F, Lee MM, et al. Exosome secreted by MSC reduces myocardial ischemia/reperfusion injury. Stem Cell Res 2010;4:214-22.

53. Bruno S, Tapparo M, Collino F, et al. Renal Regenerative Potential of Different Extracellular Vesicle Populations Derived from Bone Marrow Mesenchymal Stromal Cells. Tissue Eng Part A 2017;23:1262-73.

54. Collino F, Bruno S, Incarnato D, et al. AKI Recovery Induced by Mesenchymal Stromal Cell-Derived Extracellular Vesicles Carrying MicroRNAs. J Am Soc Nephrol 2015;26:2349-60.

55. Kholia S, Ranghino A, Garnieri P, et al. Extracellular vesicles as new players in angiogenesis. Vascul Pharmacol 2016;86:64-70.

56. Gai C, Carpanetto A, Deregibus MC, et al. Extracellular vesicle-mediated modulation of angiogenesis. Histol Histopathol 2016;31:379-91.

57. Zhang Y, Kim MS, Jia B, et al. Hypothalamic stem cells control ageing speed partly through exosomal miRNAs. Nature 2017;548:52-7.

58. Olivieri F, Albertini MC, Orciani M, et al. DNA damage response (DDR) and senescence: shuttled inflammamiRNAs on the stage of inflamm-aging. Oncotarget 2015;6:35509-21.

59. Urbanelli L, Buratta S, Sagini K, et al. Extracellular Vesicles as New Players in Cellular Senescence. Int J Mol Sci 2016;17. pii: E1408.

60. Xu D, Tahara H. The role of exosomes and microRNAs in senescence and aging. Adv Drug Deliv Rev 2013;65:368-75.

61. Figueroa JM, Skog J, Akers J, et al. Detection of wild-type EGFR amplification and EGFRvIII mutation in CSFderived extracellular vesicles of glioblastoma patients. Neuro Oncol 2017;19:1494-502.

62. Rak J, Guha A. Extracellular vesicles--vehicles that spread cancer genes. Bioessays 2012;34:489-97.

63. Melo SA, Luecke LB, Kahlert C, et al. Glypican-1

doi: $10.21037 /$ sci.2017.12.03

Cite this article as: Robbins PD. Extracellular vesicles and aging. Stem Cell Investig 2017;4:98. identifies cancer exosomes and detects early pancreatic cancer. Nature 2015;523:177-82.

64. Yang C, Kim SH, Bianco NR, et al. Tumor-derived exosomes confer antigen-specific immunosuppression in a murine delayed-type hypersensitivity model. PLoS One 2011;6:e22517.

65. Yang C, Robbins PD. The roles of tumor-derived exosomes in cancer pathogenesis. Clin Dev Immunol 2011;2011:842849.

66. Yang C, Ruffner MA, Kim SH, et al. Plasma-derived MHC class II+ exosomes from tumor-bearing mice suppress tumor antigen-specific immune responses. Eur J Immunol 2012;42:1778-84.

67. Kim SH, Bianco NR, Shufesky WJ, et al. MHC class II+ exosomes in plasma suppress inflammation in an antigenspecific and Fas ligand/Fas-dependent manner. J Immunol 2007;179:2235-41.

68. Cavallari C, Ranghino A, Tapparo M, et al. Serum-derived extracellular vesicles (EVs) impact on vascular remodeling and prevent muscle damage in acute hind limb ischemia. Sci Rep 2017;7:8180.

69. Bertoldi K, Cechinel LR, Schallenberger B, et al. Circulating extracellular vesicles in the aging process: impact of aerobic exercise. Mol Cell Biochem 2017. [Epub ahead of print].

70. Suire CN, Eitan E, Shaffer NC, et al. Walking speed decline in older adults is associated with elevated proBDNF in plasma extracellular vesicles. Exp Gerontol 2017;98:209-16.

71. Eitan E, Tosti V, Suire CN, et al. In a randomized trial in prostate cancer patients, dietary protein restriction modifies markers of leptin and insulin signaling in plasma extracellular vesicles. Aging Cell 2017;16:1430-3.

72. Eitan E, Green J, Bodogai M, et al. Age-Related Changes in Plasma Extracellular Vesicle Characteristics and Internalization by Leukocytes. Sci Rep 2017;7:1342. 